\title{
Separation of Progressive Motile Sperm from Mouse Semen Using On-chip Chemotaxis
}

\author{
Yong-Jun Ko, ${ }^{* 1}$ Joon-Ho MaEng, ${ }^{* 2}$ Byung-Chul LeE, ${ }^{* 2}$ Seungwoo LeE, ${ }^{* 1}$ Seung Yong Hwang, ${ }^{* 3 \dagger}$ \\ and Yoomin AHN*4† \\ *1 Department of Mechanical Engineering, Graduate School, Hanyang University, 222 Wangsimni-ro, \\ Seongdong-gu, Seoul 133-791, Korea \\ *2 Department of Biochemistry, Graduate School, Hanyang University, 55 Hanyangdaehak-ro, Sangnok-gu, \\ Ansan Kyeonggi-do 426-791, Korea \\ *3 Division of Molecular and Life Science, Hanyang University and GenoCheck Co. Ltd., 55 Hanyangdaehak-ro, \\ Sangnok-gu, Ansan Kyeonggi-do 426-791, Korea \\ *4 Department of Mechanical Engineering, Hanyang University, 55 Hanyangdaehak-ro, Sangnok-gu, \\ Ansan Kyeonggi-do 426-791, Korea
}

\begin{abstract}
We present a novel method for the separation of progressive motile sperm from non-progressive motile and immotile sperm. This separation was accomplished by inducing chemotaxis along a longitudinal chemical gradient in a microchip composed of a biocompatible polydimethysiloxane layer and a glass substrate. In a preliminary experiment using fluorescent rhodamine B as a marker, we verified that a chemical gradient was generated by diffusion within the microchannel. We used acetylcholine as a chemoattractant to evaluate the chemotactic response of sperm. We tested the response to a $1 / 2$ to $1 / 64$ dilution series of acetylcholine. The results of a mouse sperm chemotaxis assay showed that progressive motile sperm swam predominantly toward the outlet at an optimal chemical gradient of $0.625(\mathrm{mg} / \mathrm{ml}) / \mathrm{mm}$ of acetylcholine. This device provides a convenient, disposable, and high-throughput platform that could function as a progressive motile sperm sorter for potential use in intracytoplasmic sperm injection.
\end{abstract}

(Received August 22, 2011; Accepted October 22, 2011; Published January 10, 2012)

\section{Introduction}

The increasing rate of male infertility due to exposure to endocrine disruptors or physical stresses has recently become a serious social issue. ${ }^{1-3}$ As infertile men cannot fertilize eggs naturally, if they desire to father children their partners must be impregnated by artificial insemination. With the advent of intracytoplasmic sperm injection (ICSI), men with severe infertility are now offered the opportunity to reproduce. Prior to injecting spermatozoa, it is important to select spermatozoa that are viable, motile, and of normal head size. ${ }^{4}$ Sperm motility is the most important selection criterion for fertility. The motility of sperm is classified as progressive motile, non-progressive motile, and non-motile.,4 Non-progressive motile and non-motile sperm are considered infertile, whereas progressive motile sperm are used for ICSI.,

Different motile sperm separation techniques for ICSI, such as the swim-up method, migration-sedimentation, density gradient centrifugation, and glass wool filtration have been developed. However, these techniques have limitations including loss of

$\dagger$ To whom correspondence should be addressed.

E-mail: ahnym@hanyang.ac.kr; syhwang@hanyang.ac.kr

Y.-J. K. and J.-H. M. contributed equally.

Y.-J. K., J.-H. M., and B.-C. L. are now working at LG Innoteck, KIST, and Pharmatech. Inc., respectively. sperm DNA integrity due to the production of reactive oxygen species (ROS) and endotoxins. They are also labor- and/or time-intensive and quite expensive..$^{5,7}$ Therefore, doctors or embryologists typically use manual sorting with a microscope to separate progressive motile sperm in semen samples. Although this approach can provide convenient and fast handling compared with the techniques above, it does not provide an objective criterion for sperm separation. It is also labor-intensive and error-prone.

Microchips based on microfluidic technologies provide one of the most suitable methods for rapidly mixing and separating samples, as well as handling small amounts of sample and reagent, and integrating components for reaction and detection. With these advantages, microfluidic-based microchips have been applied to several areas of biological research such as analysis or separation of DNA, proteins, and cells. Moreover, many researchers are studying the separation or culture of living cells using microfluidic-based microchips, since the microenvironment of the microchip can be formulated to more closely resemble in vivo conditions, such as those required for fertilization and development, than conditions in a culture dish can..$^{8-10}$ Therefore, the use of microfluidic-based microchips may be a suitable method for separation of motile cells such as viable sperm.

A wide variety of methods of motile sperm separation using microfluidic-based microchips have recently been reported. ${ }^{11-16}$ These microchip-based separation techniques generally use 
active pumping by external equipment such as a syringe pump or passive pumping by hydrostatic pressure. Stable fluid flow can be prepared by controlling the dimensions of the devices using microchip-based devices. However, these methods generate laminar flow. Non-progressive motile sperms are also constantly moving randomly and can enter into the laminar flow which sorts motile sperms. Therefore, it is hard to separate only progressive motile sperm.

We present a novel microchip system that separates progressive motile sperm using chemotaxis induced by a chemical gradient of chemoattractants in microchannels. This technique does not rely on the generation of flow using an external device. Xie et al. ${ }^{17}$ confirmed the first efficiency of chemotaxis screening with a microchannel using in vitro cultured cumulus cells. In vivo, motile sperm are guided to an egg by chemotaxis, the movement of cells toward high concentrations of chemoattractant. In close proximity to the egg, sperm chemotaxis is induced by substances released from the egg. In vivo, an optimal chemoattractant concentration and chemical gradient are simultaneously formed in the oviduct. ${ }^{18-20}$ This study extends our previous efforts to replicate these in vivo conditions on a microchip with a static gradient generator, using mouse sperm as a model system, ${ }^{21,22}$ since the chemotaxis behaviors of almost all mammalian sperm are very similar. ${ }^{23,24}$ The oviduct was replaced by a microchannel filled with human tubal fluid (HTF) medium for the movement of motile sperm. Acetylcholine was used as a chemoattractant for mouse sperm. ${ }^{19}$ The major components of the microchip were a biocompatible polydimethylsiloxane (PDMS) layer and a glass substrate. Using a microscope, we observed the movement of progressive motile sperm in a radial-shaped microchannel in response to a chemical gradient generated by diffusion. We determined the optimal conditions of the chemical gradient for the separation of the progressive motile sperm from the semen sample using the microchip. We evaluated the experimental results by statistical analysis.

\section{Experimental}

\section{Sperm separation using on-chip chemotaxis}

The separation of progressive motile sperm by chemotaxis in a microfluidic-based microchip is described in Fig. S1 (Supporting Information). First, the microchannel was primed with pre-warmed HTF medium through the inlet, then $2 \mu \mathrm{L}$ acetylcholine solution was carefully dropped into the outlet (Fig. S1(a)). To maintain sperm vitality and allow diffusion of acetylcholine to generate a chemical gradient, we incubated the microchip at $37^{\circ} \mathrm{C}$ for $5 \mathrm{~min}$ (Fig. S1(b)). During this time, a chemical gradient was generated from outlet to inlet. In addition, the fluid state of the microchannel provides a static gradient environment, leading to high separation purity because there is no fluid flow. A $1-\mu \mathrm{L}$ sample of mouse semen was then carefully dropped into the inlet (Fig. S1(c)). After $10 \mathrm{~min}$, the number and motility of mouse sperm in the outlet was observed by microscopy (Fig. S1(d)).

\section{Preparation of mouse sperm}

To prepare mouse sperm, we sacrificed six $6-8$ week old mature male mice (ICR, Samtako, Inc., Korea) by cranial/ cervical dislocation. The epididymides were surgically extracted, quickly transferred to a tube containing pre-warmed HTF medium $(\mathrm{NaCl}, 593.8 \mathrm{mg} / 100 \mathrm{~mL} ; \mathrm{KCl}, 35 \mathrm{mg} / 100 \mathrm{~mL}$; $\mathrm{MgSO}_{4} \cdot 7 \mathrm{H}_{2} \mathrm{O}, \quad 4.9 \mathrm{mg} / 100 \mathrm{~mL} ; \quad \mathrm{KH}_{2} \mathrm{PO}_{4}, \quad 5.4 \mathrm{mg} / 100 \mathrm{~mL}$; $\mathrm{CaCl}_{2} \cdot 2 \mathrm{H}_{2} \mathrm{O}, 29.8 \mathrm{mg} / 100 \mathrm{~mL} ; \mathrm{NaHCO}_{3}, 210.0 \mathrm{mg} / 100 \mathrm{~mL}$; glucose, $\quad 50.0 \mathrm{mg} / 100 \mathrm{~mL}$; Na-pyruvate, $\quad 3.7 \mathrm{mg} / 100 \mathrm{~mL}$; Na-lactate, $\quad 0.34 \mathrm{mg} / 100 \mathrm{~mL}$; penicillin $\mathrm{G}, \quad 100 \mathrm{U} / \mathrm{mL}$; streptomycin, $50 \mu \mathrm{g} / \mathrm{mL}$; BSA, $4 \mathrm{mg} / \mathrm{mL} ; 0.5 \%$ phenol red $0.04 \mathrm{~mL}$; Chemicon Corp., USA), and placed in a heat block at $37^{\circ} \mathrm{C}$. Then, sperm were squeezed from the epididymis into a Petri dish containing $200 \mu \mathrm{L}$ HTF medium using a syringe needle under microscopic observation (DW-THSP, Dongwon Corp., Korea). After allowing sperm to swim out from the epididymis into the media for $5 \mathrm{~min}$, we separated epididymal tissue from the media and extracted the remaining cell debris using a micropipette. Twenty microliters of HTF media containing sperm cells were transferred to four microtubes and stored at $37^{\circ} \mathrm{C}$ in the heat block. The number of motile sperm cells including progressive and non-progressive motile sperm in each tube was measured using a hemacytometer (Superior Co., Germany). The total concentration of motile sperm was $4143 \pm 382 / \mu \mathrm{L}$.

\section{Preparation of chemoattractant}

Acetylcholine was used as a chemoattractant for the mouse sperm. To prepare the solution for the chemotaxis assay, we equilibrated acetylcholine powder (Sigma-Aldrich Corp., USA) stored at $-20^{\circ} \mathrm{C}$ at room temperature for $30 \mathrm{~min}$. This powder was then dissolved in distilled water at a concentration of $100 \mathrm{mg} / \mathrm{ml}$ and the solution was filtered using a $0.22-\mu \mathrm{m}$ syringe filter (Millipore Corp., USA). A $10-\mu \mathrm{L}$ sample of sterilized acetylcholine solution was sequentially diluted to produce $1 / 2$, $1 / 4,1 / 8,1 / 16,1 / 32$, and $1 / 64$ dilutions, which were stored at $4{ }^{\circ} \mathrm{C}$.

\section{Microchip fabrication}

The microchip was made of PDMS and glass because both substrates are biocompatible to sperm cells. PDMS is widely used in the fabrication of microchips because it has many advantages, including low cost, good optical properties, and efficient mass production. To create the microchannel, inlet, and outlet in the PDMS layer, we used a micromolding technique. First, a negative photoresist (SU-8 3035, MicroChem Corp., USA) master was made on the silicon wafer by photolithography. The height of the microchannel in the master was measured with an alpha step profiler (Nanospec AFT/200, KLA-Tencor Corp., USA). The height averaged $98.3 \pm 3 \mu \mathrm{m}$ for nine measurements of the upper, middle, and lower side. The silicone elastomer kit (Sylgard ${ }^{\circledR} 184$, Dow Corning Corp., USA) that we used to make the microchip consisted of two materials, a polymer base and a curing agent, that were mixed at a 10:1 ratio. A mixture of PDMS and curing agent was poured on the master and then the system was hardened in the oven. After $4 \mathrm{~h}$, cured PDMS layers were separated from the mold. One inlet and eight outlets were made by mechanical punching the PDMS. Lastly, the PDMS layer and glass substrate were permanently bonded together by exposure to air plasma using a plasma cleaner (PDC-32G-2, Harrick Scientific Corp., USA).

The microchip used to separate sperm by chemotaxis is shown in Fig. 1 and Fig. S2 (Supporting Information). The major components of the microchip were a biocompatible PDMS layer and a glass substrate $(28 \times 28 \times 2 \mathrm{~mm}$ and $45 \times 70 \times 1 \mathrm{~mm}$, respectively). To determine the optimal chemical gradient of chemoattractant, eight radial shaped microchannels were designed in the PDMS layer. We could put various concentrations of attractants at eight outlets and obtain results for each concentration at once. The diameter of the inlet for the semen sample reservoir was adjusted to $4 \mathrm{~mm}$. The length, width, and depth of the microchannels were $10 \mathrm{~mm}, 500 \mu \mathrm{m}$, and $100 \mu \mathrm{m}$, respectively. The diameter of each outlet for the chemoattractant 


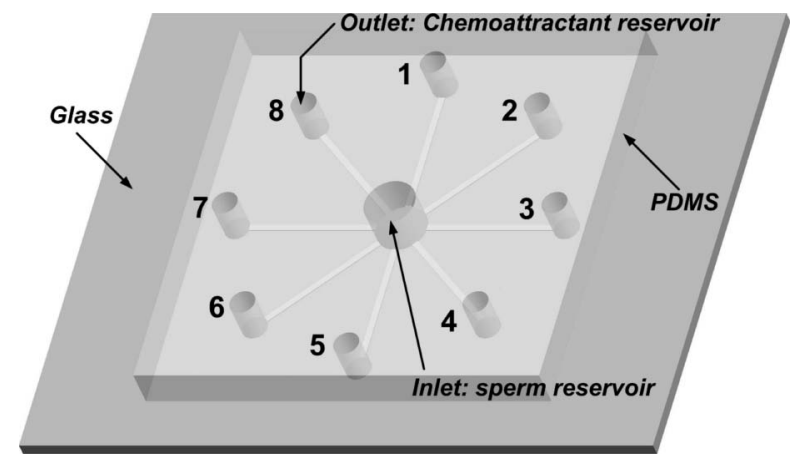

Fig. 1 Design of microchip for sperm chemotaxis assay. A number was given to each outlet well clockwise.

reservoir was adjusted to $2 \mathrm{~mm}$. The design of the microchip allowed a concentration gradient of the chemoattractant to be generated in the microchannels. The linearity of the microchannel also restricted the directions in which the sperm could swim.

Fluorescence experiment for verifying the chemical gradient in the microchannel

To confirm that a chemical gradient was created by diffusion, we measured the fluorescence intensity of rhodamine $\mathrm{B}\left(M_{\mathrm{W}}\right.$ 479.01, Fluka ${ }^{\circledR}$, UK), which has a molecular weight similar to that of acetylcholine, in the microchannel. The diffusion coefficients $(D)$ for rhodamine B and acetylcholine are defined by Eq. (1):

$$
D=\frac{k T}{6 \pi \eta a}
$$

where $k, T$, and $\eta$, are the Boltzmann constant, the absolute temperature and the viscosity of the fluid the particle, respectively, and $a$, approximately the square root of the molecular weight, is the effective size of the particle. ${ }^{25,26}$ According to this equation, the diffusion coefficients of rhodamine $\mathrm{B}$ and acetylcholine in aqueous solution at $37^{\circ} \mathrm{C}$ were $5.71 \times 10^{-10}$ and $4.132 \times 10^{-10} \mathrm{~m}^{2} / \mathrm{s}$, respectively. Since the diffusion coefficient values of the two molecules were very similar, rhodamine B could be used in the preliminary diffusion experiment as a substitute for acetylcholine.

The fluorescence experiment using rhodamine B was performed as follows. First, $40 \mu \mathrm{L}$ HTF medium was introduced into the fabricated microchip; then $2 \mu \mathrm{L}$ rhodamine $\mathrm{B}$ was carefully dropped, not injected, into the outlet of the microchip by micropipette to allow diffusion and to minimize the effect on the flow. Because of the attraction force resulting from the cohesion of the HTF solution, the droplet of rhodamine B quickly merged with HTF in the outlet of the microchip. The microchip was placed in a dry bath kept at $37^{\circ} \mathrm{C}$ for 5 and $20 \mathrm{~min}$. The microchannel was partitioned into five areas, and then the fluorescence intensity of each area was measured by confocal laser scanning microscopy (TCS SL, Leica, Germany). The absorption and the emission wavelengths were 540 and $633 \mathrm{~nm}$, respectively. The measured fluorescent intensity values were then calibrated using Leica LCS software (Leica Microsystems, Germany) system.

\section{Chemotaxis assay in the microchip}

Based on the fluorescence results, $2 \mu \mathrm{L}$ of distilled water (DW), $100 \mathrm{mg} / \mathrm{ml}$ of acetylcholine solution, and 1/2, 1/4, 1/8,

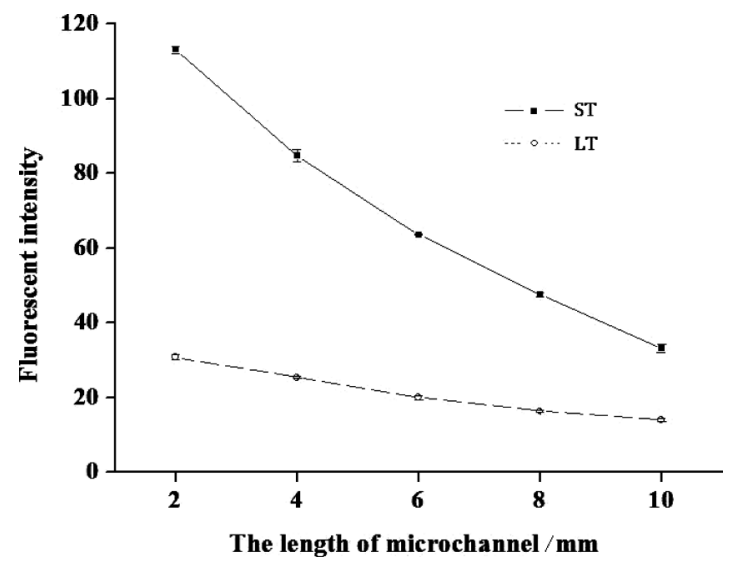

Fig. 2 Profiles of fluorescence gradients resulting from diffusion of rhodamine $\mathrm{B}$ in the microchannel. The $\mathrm{X}$-axis represents distance from the outlet. The fluorescence experiment was carried out three times.

$1 / 16,1 / 32$ and 1/64 acetylcholine dilution series were carefully dropped into each outlet; each mixture was incubated for $5 \mathrm{~min}$ prior to addition of $1 \mu \mathrm{L}$ of mouse semen sample into the inlet. After $10 \mathrm{~min}$, motile and progressive sperm that reached the outlet were counted under a microscope using a cell counter. Six mice were used for the chemotaxis experiment, and 20 sperm samples were prepared.

To evaluate the specificity of the chemotactic response of mouse sperm, we prepared three microchips; a number was assigned to each outlet, as shown in Fig. 1. In the first case, $2 \mu \mathrm{L}$ DW was dropped into every outlet; in the second case $2 \mu \mathrm{L}$ acetylcholine solution diluted at a $1 / 16$ ratio was dropped into only outlet No. 5, with DW in the other outlets; in the third case, $2 \mu \mathrm{L}$ DW was dropped into only outlet No. 5 and $2 \mu \mathrm{L}$ acetylcholine solution diluted at a 1/16 ratio was dropped into every other outlet. After the microchips were prepared, a 1- $\mu \mathrm{L}$ sperm sample was dropped into the inlet and sperm that swam into the outlet were counted.

\section{Results and Discussion}

\section{Preliminary fluorescence diffusion experiment}

Prior to the sperm separation experiment, we performed fluorescence experiments to verify that a longitudinal chemical gradient was generated in the microchannel. Figure 2 shows the chemical gradient of rhodamine B generated by diffusion in the microchannel at 5 min (marked with ST) and 10 min (marked with LT). As shown in Fig. 2, rhodamine B dropped in the outlet spontaneously diffused toward the inlet within $5 \mathrm{~min}$, and the fluorescence intensities of each area of the microchannel decreased from outlet to inlet. However, after 5 min the slope for fluorescence intensity gradually flattened. These results indicate that acetylcholine, with a diffusion coefficient similar to rhodamine $\mathrm{B}$, can be used as a chemoattractant. Also, the microchannel design of the proposed microchip provides a chemical gradient by diffusion within $5 \mathrm{~min}$. However, the gradient will change gradually and become smaller as time goes on. This can affect the movement of progressive motile sperms. In vivo, the chemical gradient should be more irregular and nonlinear than that in a microchannel chip. Even in this environment, sperms advance to an egg. Therefore, it is thought that, since circumstances in a microchip are relatively better, 


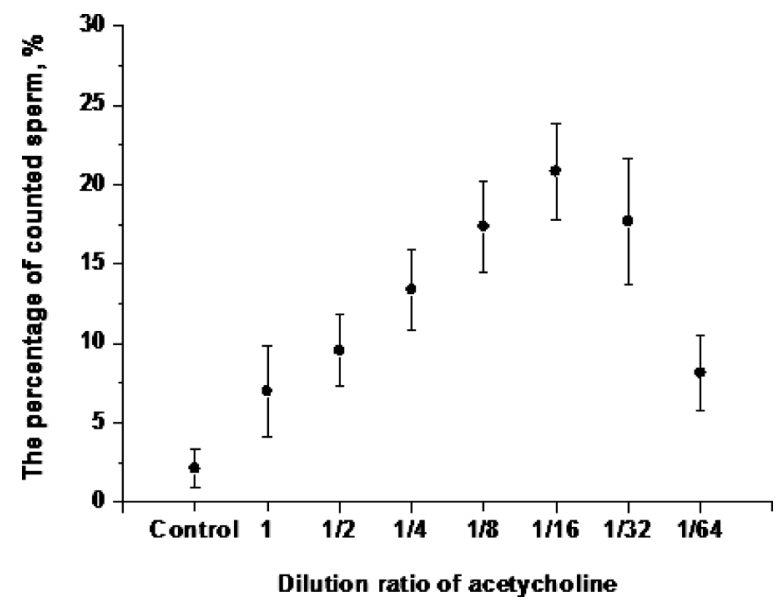

Fig. 3 Comparison of the percentage of progressive motile sperm separated by chemical gradient according to the dilution ratios of acetylcholine. The Y-axis is the percentage of the total number of sperm in the outlet. Each value shows standard errors of the mean $(n=20)(P<0.01)$.

the change of gradient after 5 min incubation is not a major obstacle for the progressive motile sperms to move.

\section{Chemotactic response of motile sperm in the microchip}

The optimal chemical gradient was determined using a dilution series of acetylcholine solutions and the radial microchip. Figure 3 shows the percentage of sperm that were counted in the outlet. As the dilution ratios increase, the number of progressive sperm that swam towards the outlet gradually increased, with the strongest response to the chemical gradient created by a $1 / 16$ dilution. Furthermore, because of a lack of fluid flow, non-progressive and immotile sperm and cell debris were observed in the inlet. We observed that fewer than $10 \%$ of the sperm swam toward the outlet at dilution ratios of $1,1 / 2$, and $1 / 64$, indicating that these extreme chemical gradient conditions negatively affected sperm mobility. When distilled water was dropped as a control, less than $3 \%$ of the sperm were observed in the outlet. This result indicated that an optimal chemical gradient was required to induce migration of progressive motile sperm. The chemical gradient created by a $1 / 16$ dilution, corresponding to $0.625(\mathrm{mg} / \mathrm{ml}) / \mathrm{mm}$ (concentration per unit length), was the optimal condition.

To further evaluate the capability of our microchip to separate progressive motile sperm by a chemical gradient, we performed statistical analysis using SAS (Ver. 9.1; SAS Institute Inc., USA). We investigated the statistical significance of the differences using Student's $t$-test at the $1 \%$ confidence level between DW and the acetylcholine dilution series, as well as the differences between a dilution factor of $1 / 16$ and the other dilution ratios. Twenty sperm samples from six mice were used. The results showed a statistically significant difference between DW and the acetylcholine dilution series with $p$-value less than 0.01 . Moreover, the number of sperm counted at a $1 / 16$ dilution was significantly different from that of the other dilution ratios $(p<0.01)$. The results of statistical analyses indicated that the chemical gradient generated in the microchip had a significant effect on sperm mobility. The progressive sperm tended to swim towards the outlet as a response to the specific chemical gradient. Furthermore, we found that a chemical gradient generated by a $1 / 16$ dilution of $100 \mathrm{mg} / \mathrm{ml}$ acetylcholine was the optimal condition.

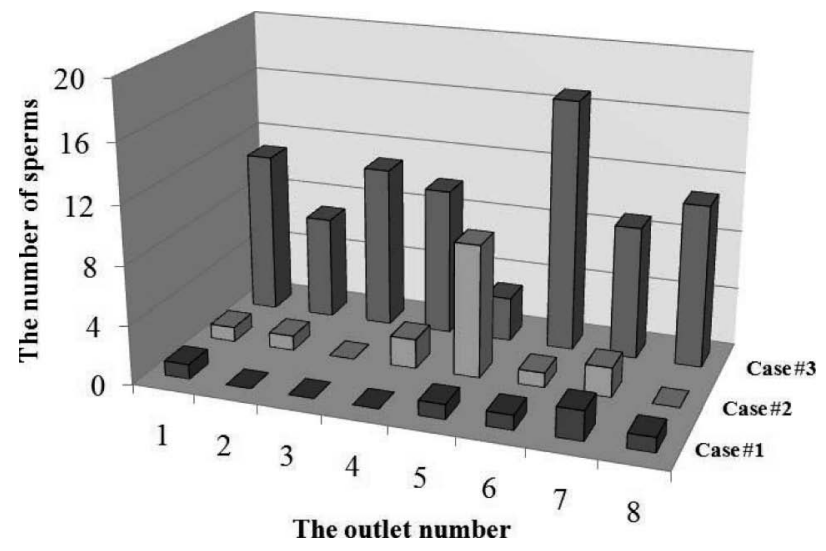

Fig. 4 The specificity test using three experimental cases. The Y-axis represents the number of progressive sperm observed at each outlet in the three cases.

We also tested the specificity of the chemotactic response of the mouse sperm in three experimental cases (Fig. 4). In the first case, although motile sperm were observed in the inlet, most of the sperm did not swim toward the outlet except through random mobility. In the second case, progressive motile sperm were observed only in outlet No. 5, and in the third case, they were observed in every outlet except for outlet No. 5. These results suggest that progressive motile sperm specifically responded to the chemical gradient generated by a $1 / 16$ dilution of the acetylcholine solution. The purity of sorted sperm samples were almost $100 \%$. Non-progressive motile sperm, dead sperm, and debris were not able to move so far as the outlets because there is no flow of media in the micro-sorting system. Sometimes non-progressive motile sperms entered the channel accidently, but it was rare that they went into an outlet during a given time period. The result shows that this method is different from other conventional methods. Conventional methods such as swim-up method separate whole motile sperms including non-progressive motile sperms, but this new chip-based method separates only progressive motile sperms.

\section{Sperm-tracking experiment}

To identify the swimming pattern of the progressive motile sperm response to a chemical gradient in the microchannel, we continued tracking one of the most progressive motile spermatozoa that swam toward the outlet under the microscope. Figure 5 shows photographs of a spermatozoon swimming toward the outlet under a chemical gradient. After generating a chemical gradient with a 1/16 dilution of acetylcholine solution, a mouse semen sample was dropped into the inlet. As shown in Fig. 5(a), most of the sperm initially moved randomly around the inlet and inlet junction. After $30 \mathrm{~s}$, chemotactically-responding sperm progressively swam toward the outlet at a mean velocity of approximately $20.8 \mu \mathrm{m} / \mathrm{s}$, and the sperm reached the outlet after approximately $8 \mathrm{~min}$. Motile sperm with velocities between 15 and $35 \mu \mathrm{m} / \mathrm{s}$ were considered to be "medium speed" sperm, ${ }^{27,28}$ and therefore we confirmed that the spermatozoa that we observed were normal motile sperm. Under a microscope, the motility and velocity of progressive motile sperm in the inlet were observed as not significantly different from those in the outlet.

We also observed progressive and non-progressive motile sperm in the middle of the microchannel. A progressive motile spermatozoon tends to swim forward in straight lines, whereas a 


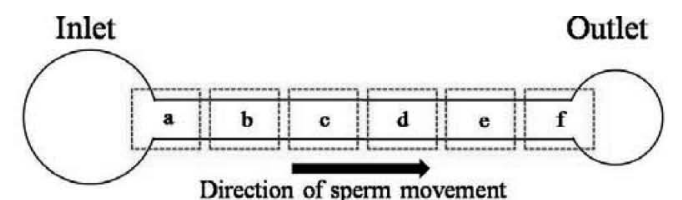

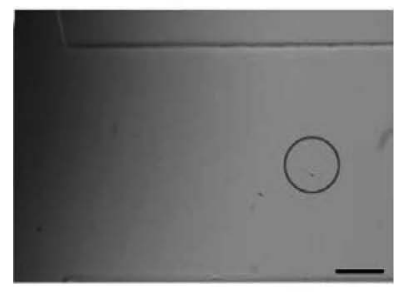

(a)

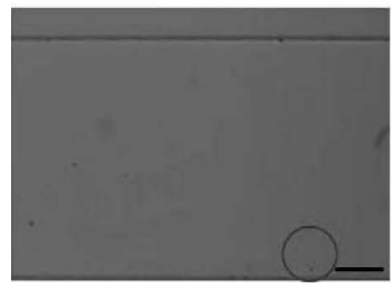

(d)

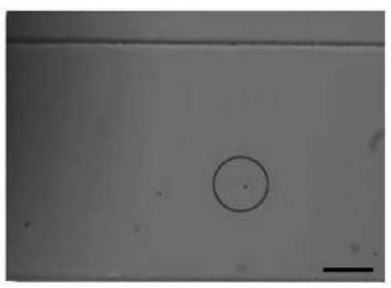

(b)

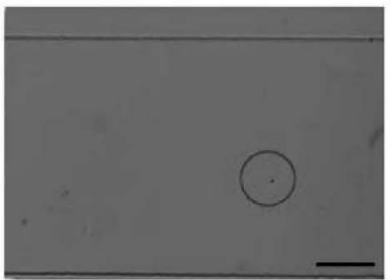

(e)

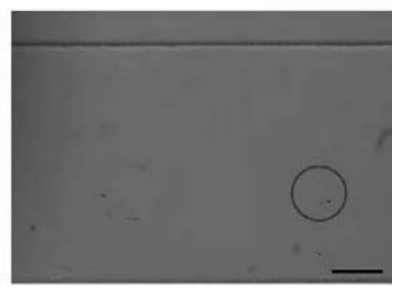

(c)

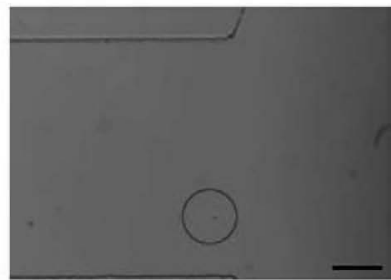

(f)

Fig. 5 Representative images of a progressive motile sperm swimming toward the outlet in response to the chemical gradient. The chemical gradient was generated from right (outlet) to left (inlet) in the microchannel, thus progressive motile sperm tended to swim from left to right. Dotted rectangular lines represent each area in the microchannel. The circle in each image indicates a single swimming spermatozoon. Scale bars are $100 \mu \mathrm{m}$.

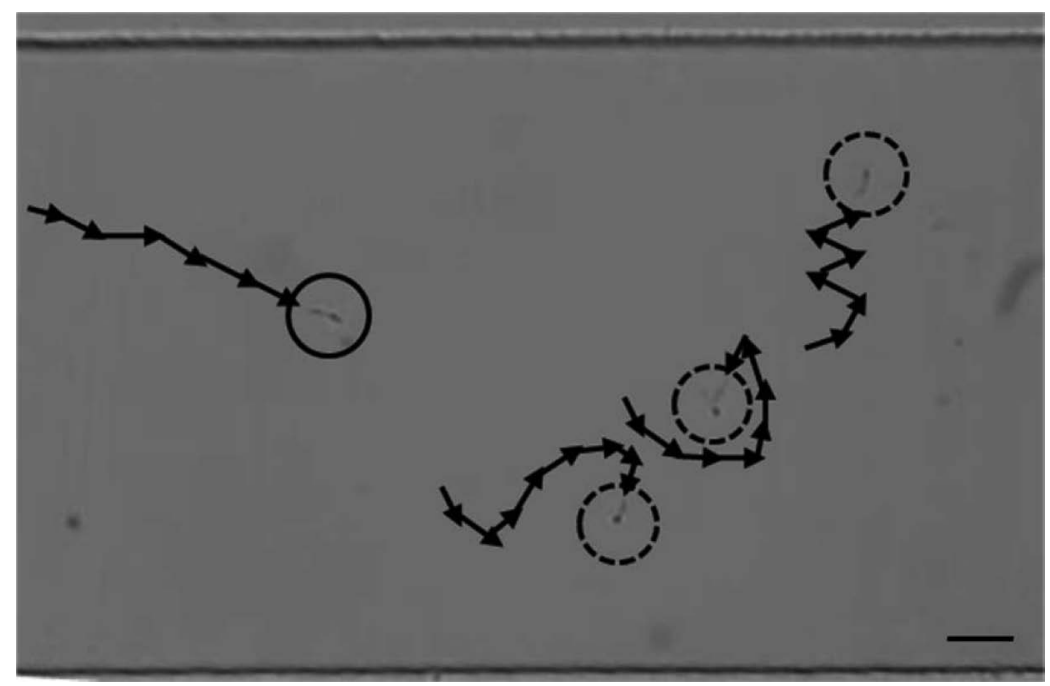

Fig. 6 Images of motile sperm track. Progressive motile sperm (solid circle) and non-progressive motile sperm (dotted circle) were observed in the microchannel. Non-progressive motile sperm tend to move in circular or random lines. Scale bar is $50 \mu \mathrm{m}$.

non-progressive motile spermatozoon tends to swim along an abnormal path, such as in tight circles, or to show random movement. ${ }^{29}$ As seen in Fig. 6, chemotactically-responding progressive motile sperm swam toward from left (inlet) to right (outlet) in straight lines. However, non-progressive motile sperm could not swim toward the outlet in spite of the chemical gradient, which indicates that we could successfully distinguish only progressive motile sperm using the microchip. On-chip in vitro fertilization (IVF) of a pig has been performed using a microfluidic device which mimics the physical conditions presents in vivo. ${ }^{30}$ In a similar way, our device can be applied to on-chip IVF. Embryos can be placed in the outlet before the sperm sorting, and then the progressive motile sperm would fertilize. After culturing for several hours on chip, fertilized eggs can be transferred to a separate culturing system. 


\section{Conclusions}

The selection of progressive motile sperm from a mixture of seminal plasma, non-reproductive cells, mature and immature spermatozoa, non-specific debris, and various microorganisms presents a significant challenge for ICSI. Published separation techniques involving conventional methods and microfluidic devices may induce DNA damage by ROS, involve labor-intensive procedures, and often yield low separation purity due to fluid flow. Here, we successfully separated progressive motile sperm using a chemical gradient of acetylcholine in a microchip without the use of external equipment. The chemical gradient was generated in the microchannel within $5 \mathrm{~min}$. There were $22 \pm 16$ progressive motile sperm per $4143 \pm 382$ motile sperm that showed the strongest attraction under the chemical gradient with a $1 / 16$ dilution of acetylcholine corresponding to $0.625(\mathrm{mg} / \mathrm{ml}) / \mathrm{mm}$.

Our microchip provides a simple, convenient, and disposable platform for separation of motile sperm. Moreover, an important feature is that progressive motile sperm can be selectively separated in the microchip in an environment that mimics that of the female oviduct. The proposed microchip may be a useful tool for ICSI by providing an objective criterion, reaching the outlet, for sperm selection. In further studies, our group will focus on integrating cell counting and the application of separation by thermotaxis.

\section{Acknowledgements}

This work was supported by a Korea Research Foundation grant funded by the Korean Government (MOEHRD, Basic Research Promotion Fund) (KRF-2006-311-D00326).

\section{Supporting Information}

The microchip technique for separation of progressive motile sperm is represented schematically in Fig. S1 and photograph of fabricated microchip is shown in Fig. S2. This material is available free of charge on the Web at http://www.jsac.or.jp/ analsci/.

\section{References}

1. A. T. Fidler and J. Bernstein, Public Health Rep., 1999, $114,494$.

2. E. Lammarrone, R. Balet, A. M. Lower, C. Gillott, and J. G. Grudzinskas, Best Pract. Res. Clin. Obstet. Gynaecol., 2003, 17, 211.

3. M. A. Szczygiel, H. Kusakabe, R. Yanagimachi, and D. G. Whittingham, Biol. Reprod., 2002, 67, 1278.

4. T. El-Toukhy and P. Braude, Curr. Obstet. Gynaecol., 2002, $12,276$.
5. R. R. Henkel and W.-B. Schill, Reprod. Biol. Endocrinol., 2003, $1,108$.

6. G. Verheyen, H. Tournaye, C. Staessen, A. De Vos, M. Vandervorst, and A. Van Steirteghem, Human. Reprod., 1999, 14, 2313.

7. S. C. Sikka, M. Rajasekaran, and W. J. G. Hellstrom, J. Androl., 1995, 16, 464.

8. R. Baudoin, A. Corlu, L. Griscom, and C. Legallais, and E. Leclerc, Toxicol. in Vitro, 2007, 21, 535.

9. J. El-Ali, P. K. Sorger, and K. F. Jensen, Nature, 2006, 442, 403.

10. Y.-C. Toh, C. Zhang, J. Zhang, Y. M. Khong, S. Chang, V. D. Samper, V. D. Noort, D. W. Hutmacher, and H. Yu, Lab Chip, 2007, 7, 302.

11. K. M. Horsman, S. L. R. Barker, J. P. Ferrance, K. A. Forrest, K. A. Koen, and K. A. Landers, Anal. Chem., 2005, 77, 742 .

12. B. S. Cho, T. G. Schuster, X. Zhu, D. Chang, G. D. Smith, and S. Takayama, Anal. Chem., 2003, 75, 1671.

13. L. J. Kricka, I. Faro, S. Heyner, W. T. Garside, G. Fitzpatrick, G. McKinnon, J. Ho, and P. J. Wilding, Pharm. Biomed. Anal., 1997, 15, 1443.

14. S. Koyama, D. Amarle, H. A. Soini, M. V. Novotny, and S. C. Jacobson, Anal. Chem., 2006, 78, 3354.

15. L. Björndahl, J. Kirkman-Brown, G. Hart, S. Rattle, and C. L. R. Barratt, Human. Reprod., 2006, 21, 145.

16. D.-B. Seo, Y. Agca, Z. C. Feng, and J. K. Critser, Microfluid Nanofluid, 2007, 3, 561.

17. L. Xie, R. Ma, C. Han, K. Su, Q. Zhang, T. Qiu, L. Wang, G. Huang, J. Qiao, J. Wang, and J. Cheng, Clin. Chem., 2010, 56, 1270.

18. M. Eisenbach, Reproduction, 1999, 4, 56.

19. M. Eisenbach and L. C. Giojalas, Nat. Rev. Mol. Cell Biol., 2006, 7, 276.

20. U. B. Kaupp, N. D. Kashikar, and I. Weyand, Annu. Rev. Physiol., 2008, 70, 93.

21. Y. J. Ko, B.-C, Lee, J.-H. Maeng, S. Y. Hwang, and Y. Ahn, in Proceedings of $\mu$ TAS, 2007, Paris, 2, 1324.

22. Y. J. Ko, J.-H. Maeng, Y. Ahn, and S. Y. Hwang, Trans. of the $K S M E(A), \mathbf{2 0 0 8}, 32,1115$.

23. E. Neuber and R. D. Powers, Human. Reprod., 2000, 15, 171.

24. F. Sun, L. C. Giojalas, R. A. Rovasio, I. Tur-Kaspa, R. Sanchez, and M. Eisenbach, Dev. Biol., 2003, 255, 423.

25. C. T. Culbertson, S. C. Jacobson, and J. M. Ramsey, Talanta, 2002, 56, 365.

26. J. P. Brody, P. Yager, R. E. Goldstein, and R. H. Austin, Biophys. J., 1996, 71, 3430.

27. M. d. C. Lopez-Garcia, R. L. Monson, K. Haubert, M. B. Wheeler, and D. J. Beebe, Biomed. Microdevices, 2008, 10, 709.

28. M. G. Herreros, I. M. Aparicio, I. Núňez, L. J. García-Marín, M. C. Gil, and F. J. P. Vega, Theriogenology, 2005, 63, 795

29. S. Tessler and P. Olds-Clarke, J. Androl., 1985, 6, 35.

30. S. G. Clark, K. Haubert, D. J. Beebe, C. E. Ferguson, and M. B. Wheeler, Lab Chip, 2005, 5, 1229. 